\title{
Incidencia de actividades de rebote en la motivación y desempeño facilitado por Liveworksheets
}

\author{
Incidence of rebound activities on motivation and performance facilitated by Liveworksheets
}

Incidência de atividades de rebote na motivação e desempenho facilitado por Liveworksheets

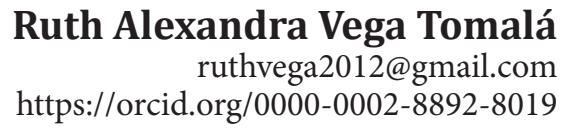

Universidad de Guayaquil, Guayaquil-Ecuador

\author{
Johnny Hiller Sigüencia Carrión \\ jsiguencia@gmail.com \\ https://orcid.org/0000-0002-4382-4021 \\ Universidad de Guayaquil, Guayaquil-Ecuador
}

Artículo recibido en junio 2021, revisado en julio 2021, arbitrado en agosto 2021 y publicado en septiembre 2021

RESUMEN

Las TIC cumplen un rol fundamental en la generación de contenidos digitales. El objetivo del presente trabajo estuvo orientado a motivar y reforzar el aprendizaje de los estudiantes a través de Liveworksheets. El estudio abordó una muestra de 20 estudiantes de primer ciclo de un Instituto Superior en la ciudad de Guayaquil. Se aplicaron encuestas, test de inicio y final. Algunos estudios relacionados con el uso de la tecnología en ambientes virtuales se han revisado, pero ninguno ha abordado el uso de Liveworksheets en actividades de rebote. La investigación fue apoyada por un análisis de datos cualitativo y cuantitativo. Los hallazgos indicaron el grado de incidencia de actividades de rebote en la motivación de los estudiantes y en su desempeño en un gran alcance. Se evidenciaron las perspectivas positivas de los estudiantes en su progreso. La investigación contribuye al campo de la enseñanza de Informática como un mecanismo formativo.

Palabras clave: Tecnología; Rebote; Liveworksheets; Motivación, Desempeño

ABSTRACT

RESUMO

ICTs play a fundamental role in the generation of digital content. The objective of this work was aimed at motivating and reinforcing student learning through Liveworksheets. The study approached a sample of 20 firstcycle students from a Higher Institute in the city of Guayaquil. Surveys, pre and post tests were applied. Some studies related to the use of technology in virtual environments have been reviewed, but none have addressed the use of Liveworksheets in bounce activities. The research was supported by qualitative and quantitative data analysis. The findings indicated the degree of incidence of rebound activities on the motivation of the students and on their performance to a great extent. The positive perspectives of the students in their progress were evidenced. The research contributes to the field of Computer Science teaching as a formative mechanism.

Key words: Technology; Rebound; Liveworksheets; Motivation, Performance
As TICs desempenham um papel fundamental na geração de conteúdo digital. O objetivo do presente trabalho foi motivar e reforçar aprendizagem dos alunos por meio de Liveworksheets. O estudo abordou uma amostra de 20 alunos do primeiro ciclo de um Instituto Superior da cidade de Guayaquil. Inquéritos, pré e pós-testes foram aplicados. Alguns estudos relacionados ao uso de tecnologia em ambientes virtuais foram revisados, mas nenhum abordou o uso de Liveworksheets em atividades de salto. A pesquisa apoiouse na análise qualitativa e quantitativa dos dados. Os achados indicaram o grau de incidência das atividades rebote na motivação dos alunos e em seu desempenho em grande parte. As perspectivas positivas dos alunos em seu progresso foram evidenciadas. A pesquisa contribui para o campo do ensino da Ciência da Computação como mecanismo formativo.

Palavras-chave: Tecnologia; Ricochete; Liveworksheets; Motivação, Desempenho 


\section{INTRODUCCIÓN}

Las Tecnologías de la Información y la Comunicación facilitan la creación y el acceso a nuevos tipos de materiales didácticos que están provocando una revisión global en la producción de contenidos y en las metodologías a aplicar en el aula (Campión et al, 2017).

Las TIC pueden potencializar en el estudiante, un dominio conceptual y un liderazgo y/o autonomía sobre su propio conocimiento, generando actitudes competenciales que mantienen su motivación hacia el alcance de las metas (Steffanell De León, y Acevedo Benavides, 2019). El rol del docente es aplicar diversas estrategias para potenciar esas habilidades.

En el contexto actual muchos docentes hacen su esfuerzo por aplicar diversas herramientas que mantengan el interés del estudiante que los motive a aprender y mantenerse interesados en un ambiente virtual. Sin embargo, factores varios como la monotonía, el cansancio y la insuficiente creatividad generan desinterés y muchas veces desmotivación de los estudiantes en aprender nuevos contenidos.

Se estudió el impacto de la herramienta Liveworksheets en la motivación y desempeño de los estudiantes a través de un análisis cualitativo y cuantitativo de datos. Por otra parte, las actividades de rebote de acuerdo a la metodología PACIE en ambientes virtuales permiten que el estudiante ejecute las actividades de refuerzo varias veces hasta que obtengan un desempeño aceptable para ellos.

Esto se denomina autorregulación ejercida por el estudiante a través de una herramienta interactiva que le dé un feedback inmediato, el estudiante promueve y autorregula su propio aprendizaje. De tal forma que el rol del docente es el de gestor del conocimiento facilitador y el rol del estudiante es el de regulador de su propio conocimiento. En este estudio se aborda al mejoramiento de la motivación y desempeño del estudiante en actividades de rebote. Es esa es la brecha o el Gap del conocimiento que se va a abordar y lo innovador de este trabajo de investigación, lo que otros trabajos de investigación no tienen.

La problemática radica en la insuficiente motivación por la que atraviesan los estudiantes por diversos factores, así como la poca innovación de los procesos de clases monótonas y sin esa insuficiente chispa de la interacción tecnológica definitivamente desencadena en una rutina en los procesos de enseñanza y aprendizaje que a su vez afecta al rendimiento de los estudiantes e incluso en muchos casos provoca la deserción (García y Tejedor, 2017).

En ese sentido, para la investigación de la problemática se plantean dos preguntas de la investigación para el presente estudio, las cuales se detallan: (a) ¿De qué manera las actividades de rebote facilitadas por Liveworksheets favorecen la motivación y el aprendizaje de los estudiantes?; (b) ¿en qué medida los estudiantes mejoran el rendimiento académico en la asignatura de Análisis de Sistemas?

La investigación se fundamenta en estudios anteriores relacionados con el uso de las tecnologías de la información y la comunicación realizadas a nivel nacional e internacional. Estos trabajos se relacionan con el presente estudio en algunos aspectos tales como el uso de la plataforma Liveworksheets. Sin embargo, no se han encontrado estudios que relacionen a las actividades de rebote con Liveworksheets. Por lo que se evidencia la innovación del presente trabajo de investigación.

Los estudios encontrados abordan los aspectos o las variables de este trabajo, pero por separado. Tal es así que se han encontrado trabajos que analizan a las Tecnologías de la Información y la Comunicación a nivel general en contextos diferentes al propuesto 
en el presente estudio. En otros casos, se menciona al desempeño académico relacionándolo con otras variables y otro tipo de población. Se detallan algunos trabajos relacionados:

En Ecuador, se efectúa un estudio abordado por Patiño, Álvarez, y Erazo (2020) quienes concluyen en que las posibilidades que brinda la plataforma Liveworksheets no solo es la interacción entre docentes y estudiantes, es también la motivación para el aprendizaje y la conciencia del trabajo a través del juego y la interacción que lleva a una interrelación social educativa. Lo que resulta de gran beneficio para la formación integral, favoreciendo el desarrollo de destrezas imprescindibles en su aprendizaje que fluyen naturalmente en un entorno lúdico que la plataforma interactiva proporciona por su practicidad en el manejo de las fichas interactivas que vuelven atractivas las tareas.

En Barcelona-España, Escardíbul y Mediavilla (2016) encuentran que la disponibilidad de recursos tecnológicos ya sea en el hogar o en la escuela influye positivamente el rendimiento académico, aunque el uso y abuso repetitivo de estas tecnologías desencadena en resultados negativos. Este trabajo citado se relaciona con el presente trabajo de investigación en algunos aspectos tales como el uso de herramientas web o TIC para el desarrollo de contenido digital. Pero el contraste radica en la aplicación de estos recursos en actividades de rebote.

En un estudio llevado a cabo en Texas, por Liu et al. (2011) con estudiantes de 12 años, luego de haber utilizado un entorno digital para enseñar Ciencias Experimentales, los alumnos y alumnas más motivados fueron aquellos que consiguieron incrementar en mayor proporción los resultados de sus pruebas. Esto les lleva a confirmar estadísticamente el hecho de a mayor grado de motivación, más significativa con un mayor potencial para la mejora en el rendimiento, y especialmente con la concepción percibida de la competencia de éstos en la materia.

De esta manera, si el diseño de un entorno digital se dirige a la potenciación del nivel de autoeficacia de los alumnos, además de otras dimensiones de la motivación, existe una mayor posibilidad de que éstos tengan más éxito en sus tareas puramente académicas (Liu et al., 2011). Por lo que se encuentra relación con la presente investigación en el aspecto de la motivación secundada por un entorno digital. Sin embargo, el estudio actual tiene características propias no se igualan pero que sí se relacionan con el estudio citado.

\section{La motivación como un factor clave en el aprendizaje}

Los factores afectivos juegan un papel muy importante en el proceso de enseñanza. Para motivar al estudiante, este debe contar con cierto grado de libertad para decidir sus acciones. Por ejemplo, en un juego, los jugadores tienen cierto grado de libertad para elegir qué tareas llevarán a cabo, pero en función de sus habilidades y de sus preferencias personales. (Contreras y Eguia, 2017).

Lo anteriormente expuesto se fundamenta con las ideas de Llorens et al. (2016) quienes enfatizan la incorporación de aspectos clave en la gamificación, los cuales son: diversión, motivación, autonomía, progresividad, retroalimentación inmediata y tratamiento del error (p. 26).

Es necesario aplicar estrategias para motivar. Las estrategias son acciones específicas, conductas, pasos o técnicas que pueden ser de comunicación, auto motivación para lograr superar tareas del lenguaje un poco difíciles. Estas son utilizadas por los estudiantes para enriquecer su forma de adquirir el conocimiento (Scarcella y Oxford, 1992, p. 2 como se cita en Olivares y Brenes 2021). 
Estas estrategias directas o indirectas hacen que los estudiantes desarrollen metacognición y autonomía en su aprendizaje.

Es así que Cano de Araúz (2009) afirmó "mientras más conscientes estén los estudiantes de las estrategias que utilizan para su aprendizaje, serán aprendices más efectivos y hábiles" (p. 400). Por lo que el desarrollo de estrategias directas e indirectas favorece la motivación.

Los aspectos afectivos que afectan o no a la motivación están relacionados con el desempeño académico del estudiante. Es así que los aspectos afectivos pueden fortalecer o entorpecer el desarrollo del aprendizaje por lo que para favorecer el aprendizaje se deben buscar estrategias creativas para lograr enganchar la atención y participación del estudiante.

\section{Desempeño académico}

El desempeño académico está ligado al rendimiento escolar pues integra conceptos, como el tipo de interacción, el éxito, el fracaso, la eficacia escolar y la competencia, (Cabero y Llorente, 2009). La implementación de las TIC en las aulas de clases permite mejorar el desempeño académico y comprensión de un tema, sea cual fuere la disciplina (Calello, Fritzsche, Quintar y Vio, 2014; Torres y Velásquez, 2015). Es así que:

Cuando se incorporan diferentes herramientas tecnológicas dentro de la formación educativa, los estudiantes tienen la capacidad de aprender de maneras distintas y a ritmos desiguales; sin embargo, para los procesos de aprendizaje estudiantil es necesario medir el rendimiento académico dentro de los saberes, de acuerdo a los estándares que se establecen dentro de una región. (Alcibar Monroy y Jiménez, 2018).
Por tal razón, incorporación de la tecnología en la universidad revela cambios en el proceso de formación e incremento en el rendimiento académico, dado por la adquisición de habilidades y actitudes (Pardo et al., 2020).

\section{Actividades de Rebote}

Las actividades de rebote forman parte de la metodología PACIE (Presencia, Alcance, Capacitación, Interacción, E-learning) para el diseño de contenidos digitales en entornos virtuales de aprendizaje. Es así que, La metodología PACIE aplicada en un entorno virtual de aprendizaje contribuye a alcanzar los objetivos del proceso de enseñanza aprendizaje mediante la incorporación de la TIC de forma gradual y reflexiva, aspectos considerados claves en la formación online (Basantes, Naranjo y Ojeda, 2018).

A nivel universitario en la docencia virtual, los docentes deben manejar metodologías para aplicar en un entorno virtual. A través de una metodología que conlleve pedagogía y técnicas didácticas que brinden garantía a los procesos de enseñanza y aprendizaje. En ese sentido, el autor ecuatoriano Pedro Camacho crea la metodología PACIE (Oñate, 2009). PACIE está basada en el acompañamiento y seguimiento al estudiante basado en la calidad y calidez humana; así como también la innovación de estrategias didácticas, el trabajo en equipo, el aprendizaje constructivo y colaborativo para coadyuvar un aprendizaje significativo (Basantes et al., 2018).

PACIE debe saber que todo ejercicio de evaluación está concentrado en la sección de rebote y de comprobación que tienen los bloques académicos de un aula virtual (Rodríguez, 2018). Según Martínez y Fuentes (2014), la metodología PACIE es un modelo que modifica el rol docente por una acción tutorial eficiente, quién desde un sentido 
humanizante motiva y realiza el acompañamiento necesario para disminuir los índices de deserción en los cursos de enseñanza virtual. En síntesis, PACIE busca sumar las tecnologías y situar al docente como impulsor de los procesos de aprendizaje (Núñez, 2015). Así mismo, en otro estudio, Rodríguez (2018) esboza que:

En cada uno de los bloques académicos se deberá diseñar las actividades de rebote y comprobación requeridos, no solo para realimentación del tutor, sino para evaluar las destrezas y habilidades alcanzadas por el estudiante, las cuales pueden ser categorizadas de manera cuantitativa a través de una calificación numérica o cualitativa a través de adjetivos de logros académicos.

La estructura de la metodología PACIE se centró en la creación de tres bloques: Bloque cero, bloque académico y bloque de cierre (Basantes et al., 2018). Las actividades de rebote corresponden al bloque académico de la metodología PACIE. Por lo tanto, Basantes et al. (2018) exponen lo siguiente:

La sección de rebote, constituye un filtro con actividades para compartir conocimientos de forma crítica y reflexiva a través de foros, chat, videoconferencias, blogs entre otros recursos delawebo plataforma Moodle, pues de esta manera el estudiante debe leer y asimilar la información de la sección de exposición, en caso contrario no podrá continuar con las actividades planificadas.

Los aspectos pedagógicos apuntan a que los estudiantes aprenden haciendo bajo un enfoque práctico. Por tal razón, (Rodríguez, 2018) refuerza la idea de (Basantes et al., 2018) al mencionar que:
Desde un punto de vista pedagógico la acción evaluativa apunta a una dinámica de aprendizaje que se basa en el "aprender haciendo", donde los resultados numéricos o calificativos de aprobación son reemplazados por objetos (premio, medalla, trofeo, otros) o acciones (alcanzar una meta, pasar a otro nivel, escapar de una situación, cambio temporal de rol, otros) que sea compatibles con el accionar de la metáfora (s.n.).

Por consiguiente, las actividades de rebote del presente estudio están orientadas a que los estudiantes resuelvan un conjunto de actividades de manera repetitiva antes de continuar con el proceso siguiente de enseñanza en el entorno virtual de aprendizaje. El éxito en la repetición de las actividades será el mejoramiento no solo de la motivación sino también del rendimiento académico de los estudiantes a través del proceso formativo.

\section{Liveworksheets}

Las plataformas tecnológicas interactivas son la herramienta fundamental en todo proceso de enseñanza y aprendizaje en entornos virtuales. Es así que (Torres y Cobo, 2017), en su estudio buscan explicar el rol de las tecnologías en la enseñanza, resaltando la importancia de su función en los contextos actuales. Siendo los estudiantes y docentes quienes asumen sus roles y conocen su situación frente al proceso que exige la sociedad en su formación.

Adicionalmente, en un estudio de las nuevas tecnologías (Domingo y Fuentes, 2019), sostienen que en la actualidad la información digital llega a todos los lugares de diferentes maneras. Los estudiantes saben manejar muybien las herramientas 
tecnológicas. Por medio de la aplicación de la tecnología que favorecen la adquisición de nuevos conocimientos, en el estudio llegan a conclusiones que se pueden resumir en la aceptación por parte de los docentes, ya que un buen proceso educativo requiere el uso de estrategias didácticas basadas en las TIC.

En relación a la plataforma interactiva (DelPrete y Cabero-Almenara, 2019), mencionan algunas características, tales como la de transformar hojas tradicionales de trabajo presentadas en pdf, jpg, doc, etc., en hojas interactivas con autocorrección y feedback inmediato, incluyendo multimedia en videos, sonidos, imágenes entre otras. Cabe mencionar que la plataforma tiene su versión gratuita y de interfaz amigable lo que facilita el desarrollo de contenidos digitales.

Liveworksheet pertenece a las herramientas Web 2.0. Su límite en la versión gratuita es hasta cien estudiantes porcuenta por lo que se adapta a entornos no tan numerosos. Esto podría ser una desventaja, pero es solucionable, por lo que un docente puede crearse otra cuenta para poder interactuar con otros grupos o cursos. La plataforma Liveworksheets se adapta a cualquier área de estudio, por lo que es una herramienta versátil como apoyo al proceso de enseñanza de cualquier asignatura. Su interface facilita el desarrollo de contenido digital. En el caso del presente estudio, se orientaron las actividades de rebote en la asignatura de análisis de sistemas para estudiantes adultos del ciclo 1 .

\section{MÉTODO}

Se aplicó la investigación a una muestra no aleatoria de 20 estudiantes del primer ciclo en la asignatura de Análisis de Sistemas de un Instituto Tecnológico Superior de la ciudad de Guayaquil. Se aplicaron técnicas de investigación tales como encuesta final. Así como también, pruebas de conocimiento de inicio (pre-test) y final (post test).
El instrumento de investigación relacionada a la encuesta es virtual a través de formularios de Google y constan de 3 preguntas de selección múltiple. Por otra parte, las pruebas de inicio y salida han sido diseñadas a través del recurso cuestionarios de 10 preguntas con un valor de un punto cada una sobre un total de diez sobre diez en la plataforma Moodle. Las actividades de rebote de la presente investigación han sido diseñadas con la plataforma interactiva Liveworksheets. Adicionalmente, las cinco actividades de rebote están relacionadas con la asignatura de análisis de sistemas para 20 estudiantes del ciclo 01. Descritas en la Tabla 4.

Luego de la recolección de los datos se ha efectuado un análisis estadístico cualitativo relacionado con las perspectivas motivadoras de los estudiantes las cuales se detallan en la siguiente sección en la Tabla 1. Así mismo, a través de un análisis cuantitativo se ha efectuado una comparación entre el pre y el post test o pruebas de inicio y salida y el análisis se lo ha efectuado con el programa IBM SPSS a través de estadística descriptiva con valores de media, moda, desviación estándar, máximos y mínimos.

Adicionalmente, se ha medido el tamaño del efecto o size effect a través de la aplicación de fórmulas estadísticas Cohen's D. Este proceso se o efectuó para medir el efecto entre el antes y el después en el presente estudio y que se detallan más específicamente en la fase de resultados y discusión.

\section{RESULTADOS Y DISCUSIÓN}

Para 7dar respuesta a la pregunta de la investigación (a) ¿De qué manera las actividades de rebote facilitadas por Liveworksheets favorecen la motivación y el aprendizaje de los estudiantes?, se ha efectuado un análisis cualitativo (Tabla 1) de las perspectivas de los estudiantes recogidos en el instrumento encuesta, detallado a continuación: 
Tabla 1. Perspectivas de motivación de estudiantes acerca de actividades de rebote facilitadas por Liveworksheets.

\begin{tabular}{lll}
\hline \multicolumn{1}{c}{ ID } & \multicolumn{1}{c}{ Perspectivas de motivación } & \multicolumn{1}{c}{ Mejoras en } \\
\hline 14 & Aprendí a reconocer símbolos de flujo & Diagramación \\
18 & Diferencio los símbolos de flujo & Diagramación \\
3 & Puedo definir a un Sistema y sus etapas. & Teoría \\
6 & He mejorado en la lógica de programac. & Programación \\
7 & Reconozco mejor las partes del sistema & Teoría \\
8 & Identifico los tipos de sistemas & Teoría \\
5 & He aprendido a hacer un esquema de flujo & Diagramación \\
1 & La repetición me ayudó a mejorar diagramac. & Diagramación \\
15 & Reconozco los elementos del Sistema Infor. & Teoría \\
17 & Identifico los sistemas operativos & Teoría \\
19 & Aprendí a resolver problemas en diagramas & Diagramación \\
16 & Actividades son interactivas me siento seguro & Diagramación \\
13 & Mis puntajes son inmediatos eso me motiva & Diagramación \\
2 & En el segundo intento mejoré mi puntaje & Diagramación \\
20 & La sopa de letras es muy motivadora & Diagramación \\
4 & He mejorado en la diagramación & Diagramación \\
10 & Aprendí a resolver problemas en diagramas & Diagramación \\
9 & Reconozco mejor los tipos de sistemas & Teoría \\
11 & Identifico los tipos de sistemas & Teoría \\
12 & Aprendía resolver problemas en diagramas & Teoría \\
\hline
\end{tabular}

Fuente: Datos de la investigación.

En la Tabla 1 se demuestran las perspectivas de los estudiantes en términos del desempeño y lo que ha logrado superar o aprender a través de las actividades de rebote.

Por otra parte, para dar respuesta a la pregunta (b) ¿En qué medida los estudiantes mejoran el rendimiento académico en la asignatura de Análisis de Sistemas? se ha hecho un análisis cuantitativo de datos (Tabla 2), los investigadores han hecho una tabla comparativa de la estadística descriptiva del test de inicio (pre test) y el test de salida (post test).

Tabla 2. Estadística descriptiva pre y post-tests.

\begin{tabular}{lcc}
\hline $\mathrm{N}$ & Valid & 20 \\
\hline Media & 5.70 & 8.60 \\
Moda & 6.00 & 9.00 \\
D. Standard & 0.66 & 0.50 \\
Mínimo & 5.00 & 8.00 \\
Máximo & 7.00 & 9.00 \\
\hline
\end{tabular}

Fuente: Datos de la investigación. 
En la Tabla 2 se muestran las estadísticas descriptivas relacionadas al test de inicio (pre-test) $\mathrm{y}$ test final (post test). Entiéndase por test de inicio el test que se aplicó al inicio de la investigación para recoger datos de cuánto sabían los estudiantes en temas relacionados con el análisis de sistemas. Mientras que test final o (post test) es el examen de salida o final donde se puede apreciar el mejoramiento del rendimiento académico de los estudiantes que fueron expuestos a las actividades de rebote.

Los resultados de puntajes obtenidos en el examen de salida o post test reflejan un incremento como se aprecia en la media obtenida pre-test 5.70 versus post-test 8.60. La desviación estándar refleja poca dispersión de los puntajes en relación a la media lo que significa que los puntajes obtenidos en el post test son casi uniformes con una desviación de 0.50 . Por lo tanto, se evidencia el mejoramiento en el rendimiento académico.

El resultado de la moda obtenida indica que la mitad de los estudiantes obtuvo una nota de seis sobre diez en el pre-test. Mientras que en el post test la mitad de los estudiantes obtuvo nueve sobre diez. Adicionalmente, se ha efectuado un análisis de tamaño del efecto de la investigación lo cual se detalla a continuación en la siguiente Tabla. Para exaltar la fiabilidad de la investigación, se ha medido el tamaño del efecto de la investigación. La misma que se evidenció a través del Cohen’s D (Tabla 3).

Tabla 3. Coeficiente de Cohen's d y tamaño del efecto o (effect size).

\begin{tabular}{lcc}
\hline & Pre-test & Post-test \\
\hline Media (M): & 5.70 & 8.60 \\
Desviación Standard (s): & 0.66 & 0.50 \\
Tamaño de muestra (n) & 20 & 20 \\
\hline
\end{tabular}

Note: Cohen's $d=(8.60-5.70) / s=0.92$

Fuente: Datos de la investigación.

Analizando la Tabla 3, la interpretación del Cohen's $d$, los resultados revelan un gran impacto porque de acuerdo a lo que indica (Salkind, 2012) el coeficiente general de interpretación es fuerte y notable cuando este está entre 0.6 y 0.8 . Por lo expuesto, el valor de Cohen's D es 0.92 es contrastado con el de 0.6 establecido por el autor Salkind. En consecuencia, el estudio demuestra un fuerte coeficiente e impacto. Las actividades y su tiempo de duración se evidencian a continuación (Tabla 4).

Tabla 4. Actividades de Rebote de la asignatura de Análisis de Sistemas Ciclo 01.

\begin{tabular}{lc}
\hline & Tiempo $(\mathrm{h})$ \\
\hline Funciones del analista de sist & 2 \\
Sistemas de Información & 2 \\
Ciclo de vida de un sistema & 2 \\
Símbolos de diagramación & 2 \\
Diagramas de flujo de datos & 2 \\
\hline
\end{tabular}

Fuente: Datos de la investigación. 
La Tabla 4 tiene el detalle de las actividades de rebote relacionadas con temas correspondientes a la asignatura de Análisis de Sistemas ciclo 01, ejecutadas durante el estudio con su respectivo tiempo específico.

\section{Discusión}

En análisis de resultados contrastado con las bases teóricas que rigen este trabajo de investigación conlleva a la comprobación de las interrogantes de la investigación. En relación a la motivación de los estudiantes y su influencia en el desempeño se pudo corroborar de manera cualitativa las perspectivas motivadoras de los estudiantes con una tendencia de gran aceptación de las actividades de rebote facilitadas por la plataforma Liveworksheets. Lo que comprueba de las Tecnologías de la Información y la comunicación influyeron de manera positiva a través de la plataforma interactiva Liveworksheets sobre la motivación de los estudiantes.

Por otra parte, el análisis de resultados estableció la medida en que las actividades de rebote mejoraron el desempeño académico de los estudiantes en la asignatura de análisis de sistemas. Esto se evidenció en el incremento de los puntajes en el test de salida (post-test) por lo que la pregunta $b$ de la investigación se ha comprobado de manera favorable. Los estudiantes tuvieron la oportunidad de repetir las actividades de rebote hasta lograr porcentajes aceptables de desempeño. Las tablas descritas en el capítulo anterior reflejan las estadísticas descriptivas con la correspondiente correlación.

En relación alCohen'd se puede determinar quela mayoría de las investigaciones de carácter científico se basan en la confirmación de hipótesis con el soporte de ajustar los datos aun modelo estadístico referencial a través de un análisis cuantitativo. De acuerdo a los valores referenciales de Cohen'd si la probabilidad de obtener resultados por casualidad es menor que .05 , entonces generalmente se acepta la hipótesis experimental como verdadera. En otras palabras, hay un efecto en la muestra de estudio.

Por lo general, los investigadores pueden reportar que "hay un efecto significativo de tales variables" Sin embargo, no se puede enfocar todo en un término "significativo", porque incluso podría ser considerado un resultado bajo de significación, esto no necesariamente expresa que el efecto fue importante porque pequeños efectos podrían producir resultados significativos, sobre todo al usar grandes muestras. En el caso del presente estudio de investigación, el efecto fue 0.92 que representa la confirmación de las preguntas de la investigación en escala aceptable.

\section{CONCLUSIONES}

En análisis de resultados contrastado con las bases teóricas que rigen este trabajo de investigación conlleva a la comprobación de las interrogantes de la investigación. En relación a la motivación de los estudiantes y su influencia en el desempeño se pudo corroborar de manera cualitativa las perspectivas motivadoras de los estudiantes con una tendencia de gran aceptación de las actividades de rebote facilitadas por la plataforma Liveworksheets. Lo que comprueba de las Tecnologías de la Información y la comunicación influyeron de manera positiva a través de la plataforma interactiva Liveworksheets sobre la motivación de los estudiantes.

Por otra parte, el análisis de resultados estableció la medida en que las actividades de rebote mejoraron el desempeño académico de los estudiantes en la asignatura de análisis de sistemas. Esto se evidenció en el incremento de los puntajes en el test de salida (post-test) por lo que la pregunta b de la investigación se ha comprobado de manera favorable. Los estudiantes tuvieron la 
oportunidad de repetir las actividades de rebote hasta lograr porcentajes aceptables de desempeño. Las Tablas descritas en el capítulo anterior reflejan las estadísticas descriptivas con la correspondiente correlación.

En relación alCohen'd se puede determinar quela mayoría de las investigaciones de carácter científico se basan en la confirmación de hipótesis con el soporte de ajustar los datos aun modelo estadístico referencial a través de un análisis cuantitativo. De acuerdo a los valores referenciales de Cohen'd si la probabilidad de obtener resultados por casualidad es menor que .05, entonces generalmente se acepta la hipótesis experimental como verdadera. En otras palabras, hay un efecto en la muestra de estudio.

Por lo general, los investigadores pueden reportar que "hay un efecto significativo de tales variables" Sin embargo, no se puede enfocar todo en un término "significativo", porque incluso podría ser considerado un resultado bajo de significación, esto no necesariamente expresa que el efecto fue importante porque pequeños efectos podrían producir resultados significativos, sobre todo al usar grandes muestras. En el caso del presente estudio de investigación, el efecto fue 0.92 que representa la confirmación de las preguntas de la investigación en escala aceptable.

\section{REFERENCIAS}

Alcibar, M.F, Monroy, A. y Jiménez, M. (2018). Impacto y Aprovechamiento de las Tecnologías de la Información y las Comunicaciones en la Educación Superior. Información Tecnológica, 29 (5). 101-110. doi: 10.4067/S071807642018000500101

Basantes, Andrea V.; Naranjo, Miguel E.; Ojeda, Vivian. (2018). PACIE Metodología PACIE en la Educación Virtual: una experiencia en la Universidad Técnica del Norte. Formación Universitaria, 11(2), 35-44. 10p. DOI: 10.4067/ S0718-50062018000200035
Cabero, J., y Llorente, M. (2009). Actitudes, satisfacción. rendimeinto academico $y$ comunicación on line en proxesos de formación universitaria en blended learning. Revista electrónica teoría de la educación, 10(1)

Cano de Araúz, O. (2009). Language learning strategies and its implication for second language teaching. Revista de Lenguas Modernas, 11(1), 399-411. https://revistas.ucr.ac.cr/index.php/ rlm/article/view/9454/8904

Calello, T., Fritzsche, F., Quintar, A. y Vio, M. (2015). Redes y nuevas tecnologías de información y comunicación en las asambleas vecinales de Buenos Aires. Revista Economía, Sociedad y Territorio, 4(16), 609-33

Campión, R. Santiago, V. M. Maeztu Esparza y Andía Celaya, L. (2017). Los contenidos digitales en los centros educativos: Situación actual y prospectiva. Revista Latinoamericana De Tecnología Educativa, 16(1), 51-66. doi:10.17398/1695-288X.16.1.51

Contreras Espinosa R. y Eguia j. (editores) (2017). Experiencias de gamificación en aulas. InComUAB Publicacions, 15. Bellaterra: Institut de la Comunicación, Universitat Autònoma de Barcelona. ISBN 978-84-944171-6-0

Del-Prete, A, y Cabero-Almenara, J. (2019). Las plataformas de formación virtual: algunas variables que determinan su utilización. Apertura (Guadalajara, Jal.) ,11(2), 138-153. https://doi.org/10.32870/ap.v11n2.1521

Domingo, M., y Fuentes, M. (2019). Innovación educativa: Experimentar con las Tic y reflexionar sobre su uso. [Educational innovation: Experimenting with ICT and reflecting on its use.]. Pixel-Bit. Revista de Medios y Educación, 0(36), $171-180$. Obtenido de https://n9.cl/ mc9ez

Escardíbul, J. y Mediavilla, M. (2016). El efecto de las TIC en la adquisición de competencias. Un análisis por tipo de centro educativo. Revista española de pedagogía, 264, 317-335

García, A., y Tejedor, F. (2017). Percepción de los Estudiantes sobre el valor de las TIC en sus estrategias de aprendizaje y su relación con el rendimiento. Educación XXI, 20(2), 137-159 
Liu,Min;Horton,Lucas; Olmanson, Justin;TOPRAC, Paul. A study of learning and motivation in a new media enriched environment for middle school science. Educational Technology Research and Development, v. 59, n. 2, p. 249-265, Feb. 2011

Llorens Largo, F., gallego-Durán, F. J., VillagráArnedo, C. J., Compañ, P., Satorre Cuerda, R., y Molina-Carmona, R. (2016). Gamificación del proceso de aprendizaje: lecciones aprendidas

Martínez, E. y Fuentes, M. (2014). Implementación de la metodología PACIE en cursos semipresenciales en el Entorno Virtual de Aprendizaje de TIC. Revista Iberoamericana de Producción Académica y Gestión Educativa, ISSN: 2007-8412, 2(2), 1-12 (2014)

Oñate, L. (2009). La Metodología PACIE. FATLA (Fundación para la Actualización Tecnológica de Latinoamérica). En línea: http: //bit. ly/2vQPXEA, acceso: 10 septiembre 2016

Olivares, C. y Brenes, V. (2021). Indirect Learning Strategies in University Students' EFL Development. MEXTESOL Journal, Vol. 45, No. 1, 2021. Recuperado de https://files.eric.ed.gov/ fulltext/EJ1289008.pdf

Patiño-Quizhpi, D., Álvarez-Lozano, M., y Erazo-Álvarez, J. (2020). Estrategias lúdicas para desarrollar la lecto-escritura mediante la plataforma Liveworksheets. CIENCIAMATRIA, 6(3), 408-427. https://doi.org/10.35381/ cm.v6i3.408

Pardo-Cueva, M., Chamba-Rueda, L. M., Gómez, Á. H., y Jaramillo-Campoverde, B. G. (2020). Las TIC y rendimiento académico en la educación superior: Una relación potenciada por el uso del Padlet. Revista Ibérica de Sistemas e Tecnologias de Informação, (E28), 934-944

Salkind, N.J. (2012). Statistics for People Who (Think They) Hate Statistics. New Delhi: SAGE

Steffanell De León, I. H. E., y Acevedo Benavides, M. A. (2019). Mediación TIC y su influencia en la satisfacción y desempeño académico de los estudiantes de pregrado (Doctoral dissertation, Universidad de la Costa)

Rodríguez, T. Y. (2018). Entorno virtual de aprendizaje centrado en la metáfora educativa. Universidad Ciencia Y Tecnología, 22(86), 11. Recuperado a partir de https://www.uctunexpo. autanabooks.com/index.php/uct/article/ view/175

Torres, P., y Cobo, J. (2017). Tecnología educativa y su papel en el logro de los fines de la educación. [Educational technology and its role in achieving the aims of education]. Educere, 21(68), 31-40. Recuperado de https://n9.cl/nrcdx 